\title{
An Integrated System for Radioluminescence, Thermoluminescence and Optically Stimulated Luminescence Measurements
}

\author{
Chang-Young Park', Young-Kook Park', Ki-Soo Chung ${ }^{1, *}$, Jong-Duk Lee², Jungil Lee ${ }^{3}$, Jang-Lyul Kim³ \\ ${ }^{1}$ Department of Physics and Research Institute of Natural Science, Gyeongsnag National University, Jinju, Korea; ${ }^{2}$ College of Interdisciplinary and Creative \\ Studies, Konyang University, Nonsan, Korea; ${ }^{3}$ Korea Atomic Energy Research Institute, Daejeon, Korea
}

\section{Technical Paper}

Received August 4, 2018

Revision September 29, 2018

Accepted November 5, 2018

Corresponding author: Ki-Soo Chung

Department of Physics and Research Institute of Natural Science, Gyeongsang National University, 501 Jinju-daero, Jinju 52828, Korea

Tel: $+82-55-772-1402$

Fax: +82-55-772-1409

E-mail:chungks@gnu.ac.kr

This is an Open-Access article distributed under the terms of the Creative Commons Attribution NonCommercial License (http://creativecommons.org/ licenses/by-nc/4.0) which permits unrestricted noncommercial use, distribution, and reproduction in any medium, provided the original work is properly cited.

Copyright $\odot$ 2018 The Korean Association for Radiation Protection

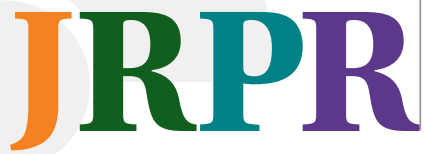

Background: This study aims to develop an integrated optical system that can simultaneously or selectively measure the signals obtained from radioluminescence (RL), thermoluminescence (TL), and optically stimulated luminescence (OSL), which are luminescence phenomena of materials stimulated by radioactivity, heat, and light, respectively. The luminescence mechanism of various materials could be investigated using the glow curves of the luminescence materials.

Materials and Methods: RL/TL/OSL integrated measuring system was equipped with a X-ray tube $(50 \mathrm{kV}, 200 \mu \mathrm{A})$ as an ionizing radiation source to irradiate the sample. The sample substrate was used as a heating source and was also designed to optically stimulate the sample material using various light sources, such as high luminous blue light emitting diode (LED) or laser. The system measured the luminescence intensity versus the amount of irradiation/stimulation on the sample for the purpose of measuring RL, TL and OSL sequentially or by selectively combining them. Optical filters were combined to minimize the interference of the stimulation light in the OSL signal. A long-pass filter ( $420 \mathrm{~nm}$ ) was used for $470 \mathrm{~nm}$ LED, an ultraviolet-pass filter (260-390 nm) was used for detecting the luminescence of the sample by PM tube.

Results and Discussion: The reliability of the system was evaluated using the RL/OSL characteristics of $\mathrm{Al}_{2} \mathrm{O}_{3}: \mathrm{C}$ and the $\mathrm{RL} / \mathrm{TL}$ characteristics of $\mathrm{LiF}: \mathrm{Mg}, \mathrm{Cu}, \mathrm{Si}$, which were used as dosimetry materials. The RL/OSL characteristics of $\mathrm{Al}_{2} \mathrm{O}_{3}: \mathrm{C}$ showed relatively linear dose-response characteristics. The glow curve of $\mathrm{LiF}: \mathrm{Mg}, \mathrm{Cu}, \mathrm{Si}$ also showed typical RL/OSL characteristics.

Conclusion: The reliability of the proposed system was verified by sequentially measuring the RL characteristics of radiation as well as the TL and OSL characteristics by concurrent thermal and optical stimulations. In this study, we developed an integrated measurement system that measures the glow curves of RL/TL/OSL using universal USB-DAQs and the control program.

Keywords: Radioluminescence, Thermoluminescence, Optically stimulated luminescence, Dosimetry, Luminescence mechanism

\section{Introduction}

Thermoluminescence (TL) and optically stimulated luminescence (OSL) are two of the most widely used dosimetry and analysis methods [1-3]. Their luminous phenomena are mainly caused by the trapping level, which is generated by the impurities or structural defects in the material or is shown in crystals with recombination center 
(RC). When the electrons trapped in the trapping level are exposed to radiation, particle beams or ultraviolet rays these electrons are combined with the holes in RC through the conduction band. Light can be illuminated in this process, where the luminescence by thermal and optical stimulations is TL and OSL, respectively. Using the luminescence intensity against the TL or OSL stimulation, the electron density in the trap or the physical properties of the trap can be estimated. Thus, it can be used in determining the energy band structure of materials or estimating the amount of radiation irradiated onto such materials [4]. On the contrary, radioluminescence (RL) is a phenomenon that light is emitted simultaneously by the recombination of the pair-produced electron and hole in the recombination center when the material is exposed to radiation [5]. This can be used in measuring radiation in real time.

While RL and TL/OSL are both similar phenomena where light is emitted with respect to the energy level of materials, they have been measured and used separately. Although Riso measuring device, which is used commercially, can measure both TL and OSL, some additional devices need to be attached to measure RL because it does not measure RL consistently under the environments, such as radioactive, thermal or optical stimuli. If measured consistently, the behavior of electrons and holes in the material from each luminescence can be analyzed multi-dimensionally and interpreted more delicately. For example, among the energy levels of a crystal, some trapping levels are not influenced by $\mathrm{RL}$, whereas all trapping levels are more significantly influenced by TL and OSL. Therefore, through the analysis of these luminescence curves obtained from RL and TL/OSL, we can obtaine more plentiful information about the trapping levels rather than the case of individual treatment.

In this study, an integrated measuring device was developed to analyze more accurately the luminescence mechanism of the given materials through the analysis of the RL and TL or OSL luminescence curves obtained by applying various stimuli to the materials. The control and measurement in the device were performed by the data acquisition system (DAQ), and LabView (ver. 2014, National Instruments, Austin, TX) was used to develop a program that can automate and control the overall device. The proposed system can perform a delicate control of the amount of radiation and heat and optical stimulation and thus, can perform various measuring tasks, such as the control of the amount of X-ray, TL measurements with different temperature in-

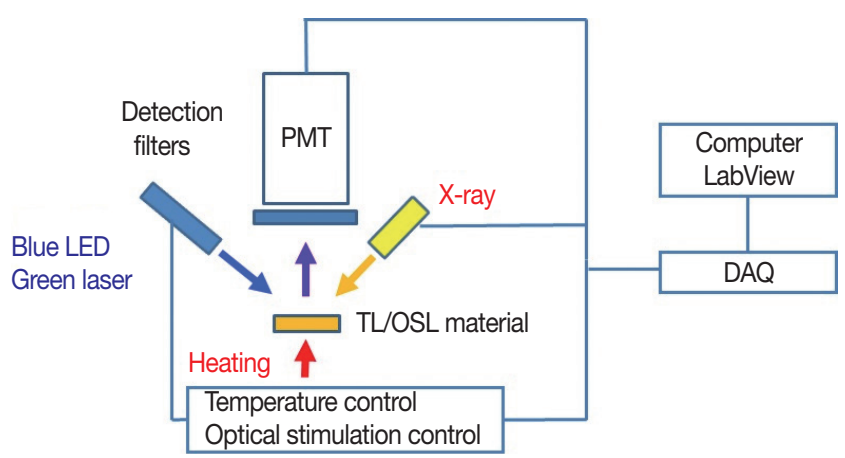

Fig. 1. Schematic layout of $R L$ and OSL measurement system.

crease ratios, OSL measurement in various measurement modes, and simultaneous measurement of RL and OSL or $\mathrm{RL}$ and TL, among others.

\section{Materials and Methods}

The configuration of the measuring devices is shown in Figure 1; an X-ray tube system was used for the ionizing radiation source. Other light sources including light emitting diode (LED) or laser were used for optical stimulation after having been exposed to irradiation. Additionally, various kinds of substrate were used as the heat source for thermal stimulation. The very weak luminescence generating from the material due to the ionizing radiation, heat and/or light stimuli could be measured using a photomultiplier tube (PMT) system. To control the X-ray system, the stimulation light source and the temperature of substrate, and to measure the amount of luminescence for thermal or optical stimulation and other environmental parameters such as temperature, the general purpose DAQ connected to a computer were used. The X-ray tube and the stimulating light source are irradiated to the sample on an inclined angle, whereas the resulting luminescence is measured vertical direction. To reduce the stray light from stimulating light being added to the measurement of the luminescence as much as possible, some specific combinations of optical filters are installed in front of both the stimulating light source and the PMT. The overall control and measurement are passed through DAQ (NI USB 6343, National Instruments, Austin, TX) and logged into and out from the computer. And LabView helps the overall measurement process to be performed consistently.

\section{Optical Stimulation Control and Optical Measurement Part}

The stimulant light applied to the sample is similar to that 
of a typical OSL measuring device. The light source is irradiated to the specimen a slope of $45^{\circ}$. Our own standard adaptors are used to attach various types of LED or laser. There are six holes to which the adapters can be inserted, these holes make a circle at a same angle. The X-ray tube is installed on one of holes and up to five light sources can be installed on the remaining holes. In this way, various combinations of light sources from LED and laser to UV LED, IR LED, and blue LED can be used. The developed system used three identical LEDs to maximize the amount of light flux and provide homogeneous irradiation. The stimulating flux was measured by the photodiode on the side of sample substrate. The current of photodiode is acquired as the flux of the optical stimulation and can also be used as a feedback signal to maintain the amount of the intensity at a required value. It can also be applied to various types, including CW-OSL (continuous wave OSL), which maintains the flux, or LM-OSL (linear modulation OSL), which increases the flux linearly.

In the case of $\mathrm{Al}_{2} \mathrm{O}_{3}: \mathrm{C}$ or $\mathrm{LiF}: \mathrm{Mg}, \mathrm{Cu}, \mathrm{Si}$, the center wavelength of the main luminescence is near to $420 \mathrm{~nm}$ with a wavelength range of 320-480 $\mathrm{nm}$. When recombination center in the band gap of such materials has holes by the ionizing radiation; light could be emitted when electrons recombine with these holes. These OSL materials mainly use a high-brightness blue LED such as Luxeon V (Lumileds, San jose, CA). The bandwidth wavelength of blue Luxeon $\mathrm{V}$ is $470 \pm 25 \mathrm{~nm}$, and its maximum current is $700 \mathrm{~mA}$. To block the wavelength of this zone from the stimulating light, a longpass filter by Schott, GG420 (Schott AG, Mainz, Germany), was used [6]. Furthermore, a $7.5 \mathrm{~mm}$-thick UV band-pass filter UG11 (Schott AG, Mainz, Germany) was installed on PMT to pass the light between 260 and $390 \mathrm{~nm}$. This filter blocks the stimulating light that passed GG420 from entering the PMT. Therefore, luminescence was measured in the wavelength range of 320 to $390 \mathrm{~nm}$. Besides, various light sources, such as $532 \mathrm{~nm}$ green laser or $250 \mathrm{~nm}$ UV LED, and corresponding optical filters could be combined.

The used PMT was ET 9235B (ET Enterprises, Uxbridge, UK) whose wavelength is between 290 and $630 \mathrm{~nm}$, the measurement bore was $48 \mathrm{~mm}$, and the yield rate was $63 \times 10^{6}$. To minimize the effect of the noise, the counting mode was used for the measurement, and toward this end, amplifier discriminator, AD6 (ET Enterprises, Uxbridge, UK), by ET Enterprises was used. In this way, the signal on the PMT is converted into standardized TTL pulses at the resolution of $20 \times 10^{6}$ per one second. These pulses are input to the coun- ter/timer channel of the DAQ to count the number of photons entering at a specific period of time.

\section{Thermal Stimulation and Sample Substrate}

In the proposed system, the sample substrate is used as a heating element. To control the temperature, the controlling voltage between 0 to $5 \mathrm{~V}$ is output via the DAQ by comparing the temperature of the sample substrate and the required temperature using the temperature controlling program. The temperature controlling device first generates a direct voltage between 0 to $300 \mathrm{~V}$ that corresponds to the controlling voltage. The wave-generating device generates a square wave at $35 \mathrm{kHz}$. And the IGBT, a power controlling device, repeats the on-off of the DC voltage with proper duty ratio. By these sequential procedure, the high-frequency controlled power at $35 \mathrm{kHz}$ is generated. Through a high-frequency converter that uses a ferrite core to reduce to a single digit voltage with high current up to $20 \mathrm{~A}$. This voltage delivers the power to the heating element. Attached under the sample substrate is a thermocouple in K-type, which measures the temperature. The thermocouple generates minute voltages based on the temperature and is connected to microprocessor-based SG-3011 (ICP DAS, Hsinchu, Taiwan) to output analog values between 0 to $10 \mathrm{~V}$ in correspondence to the sample temperature [7].

\section{X-ray Irradiation Device}

Riso device (RISO National Lab, TL/OSL DA-20, Roskilde, Denmark) uses a beta-ray source as the radiation source and irradiates radioactive rays by moving the specimen from the measurement location to the radiation generation device. On the other hand, the developed measurement device can irradiate radioactive rays and measures the TL/OSL at the same spot without moving the sample by positioning of the $\mathrm{X}$-ray tube and light source at the same arrangement. So, under the identical environment, it can apply radiation, thermal and/or optical stimulation simultaneously to the sample for the measurement. Unlike most OSL measuring devices such as Riso system, it can immediately measure the luminescence emitted at the time of the radiation, i.e., RL, under the same environment as the measurement of TL/OSL. Such measured data can be analyzed using an algorithm that interprets RL and OSL as a consistent process.

In the measurement system, the X-ray irradiation device is attached with a same standardized adapter as the stimulating light source. As explained earlier, it is installed on one of 
the six holes and irradiates the light at a $45^{\circ}$ adapter in the same specifications as the stimulating light source and Moxtek as the X-ray irradiation device $(50 \mathrm{kV}$ Cable $10 \mathrm{~W}$ with MAGNUM X-ray Source, Orem, UT). Its maximum voltage, current, and power are $50 \mathrm{kV}, 200 \mu \mathrm{A}$, and $10 \mathrm{~W}$, respectively. The target metal is $\mathrm{Pd}$ with maximum radiation of 30 $\mathrm{mGy} \cdot \mathrm{s}^{-1}$. By installing a $1 \mathrm{~mm}$-thick aluminum filter, the lower energy part below $20 \mathrm{keV}$ was blocked. At this point, the level of X-ray radiation with the attachment of the aluminum filter can be acquired using $\mathrm{Al}_{2} \mathrm{O}_{3}: \mathrm{C}$, an OSL standard dosimetric material. Using beta-ray survey device (6527B, Studsvik Co., Nyköping, Sweden) of known radiation, stimulating light of $13.98 \mathrm{mGy}$ was irradiated and OSL was measured to estimate the amount of radiation. Using the conversion factor and energy compensation factor, radiation of 2.23 $\mathrm{mGy} \cdot \mathrm{s}^{-1}$ was acquired.

The accelerating voltage and current applied to the X-ray tube can be controlled by entering two controlling voltages to the control module. To control the amount of X-ray and exposure time, DAQ generates two controlling voltages from digital-analog output channels, which are entered to the high voltage power supply which manage the accelerating voltage and current of the X-ray tube. Moreover, since the power supply outputs the actual accelerated voltage and current in two analog voltages while the X-ray irradiation device is operating, this anode voltage and electron current can be acquired by the DAQ.

\section{Construction of the Device}

As illustrated in Figure 2 for this system, the sample sub-

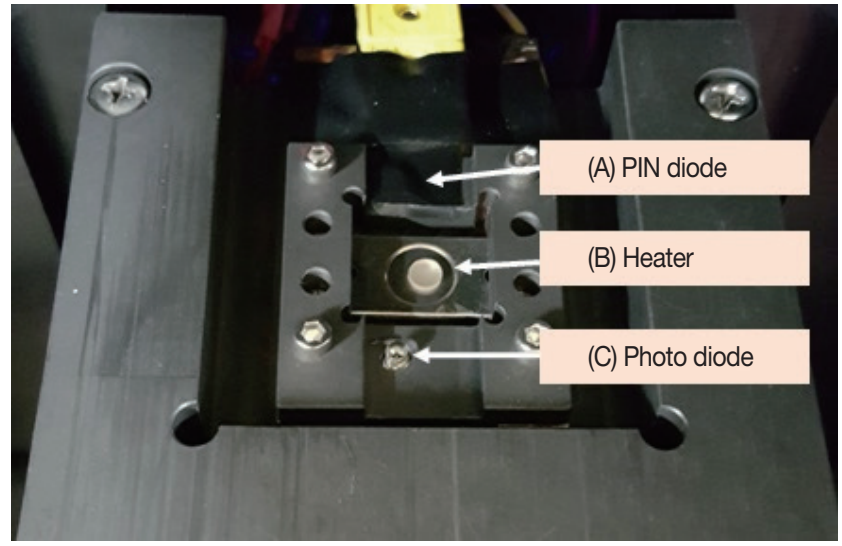

Fig. 2. Sample holder. (A) PIN diode measures the X-ray intensity, (B) Heaters and thermocouples measure the heating and temperature of the sample, and (C) Photo diode measures the intensity of the stimulating light. strate was attached with the heating element, photodiodes were used to measure the flux of the stimulating light, and PIN diodes were used to measure the intensity of the X-ray. To measure the radiation, silicon PIN photodiode (VISHAY, BPW34, Malvern, PA) was used. The luminescent signal originated in the exposure to the ionizing radiation was too weak and thus, it was measured by amplifying it using Amplifier C7319 (Hamamatsu Photonics, Shizuoka, Japan). The temperature, amount of the stimulating light, and intensity of the X-ray were measured by connecting the system to the analog voltage input channels of the DAQ. Meanwhile, without sample, the background of PMT was $\sim 10$ count per 0.1 seconds, and with the stimulating light at its maximum, it was $\sim 20$ count per 0.1 second, effectively blocking the stimulating light. When an aluminum filter is attached at $50 \mathrm{kV}, 100$ $\mu \mathrm{A}$, it will increase roughly up to $\sim 4 \times 10^{3}$ count per $0.1 \mathrm{sec}$ ond. Therefore, the estimated amount of background signal could be removed during the glow curves analysis stage.

Figure 3 is the schematic diagram of the system designed to meet the RL/TL/OSL measurement requirements. Since

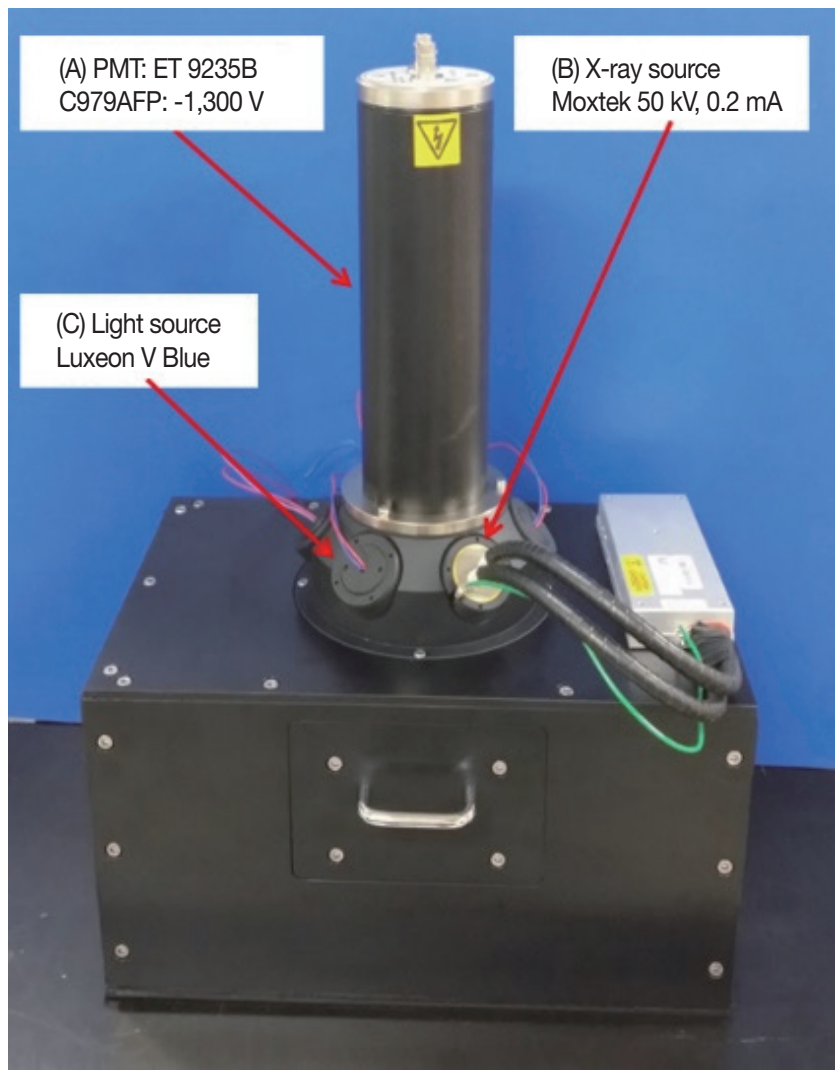

Fig. 3. RL/TL/OSL Measurements System. (A) PM tube: ET 9235B, (B) X-ray source: Moxtek 50 kV $200 \mu \mathrm{A}$, and (C) Light source: Luxeon $\vee$ Blue. 
including the X-ray source, it requires the radiation shielding for its whole body. A shielding box was made in which the $\mathrm{RL} / \mathrm{TL} / \mathrm{OSL}$ measuring device was inserted. This shielding box was made with lead, acrylic, and aluminum panels, and the X-ray source would function only under certain conditions; for example, the power of the $\mathrm{X}$-ray radiation device would be cut off if radiation is exposed outside due to certain circumstances like the opening of the door.

The system used USB-6343 (National Instruments, Austin, TX) as DAQ for measurement and control, which is a universal data acquisition device. The voltage output section of the USB-6343 with a 4-channel 16-bit digital to analog converter was used in controlling the amount of the light flux and temperature applied to the specimen, and the voltage and current of the X-ray controlling module. The voltage input section has a 32-channel 16-bit analog to digital converter, which is used to measure the flux of the stimulating light, and the voltage and current applied to the X-ray source. Furthermore, one out of the four channels of the count/timer was used to count the photons of PMT. Therefore, all control and measurement activities were conducted via USB-6343, as shown in Figure 4.

The measurement and control of the whole system were done using LabView. First, it controlled the voltage, current, and exposure time of the X-ray. Next, the thermal stimulation temperature was increased at a certain speed based on the temperature increase rate, and once it reached a certain value, it was maintained. Finally, the amount of the stimulating light was controlled to select the type of various functions, such as CW-OSL, LM-OSL measurement modes, or others. Accordingly, various radiation was selected and the temperature and stimulating light were controlled to measure the RL/TL or RL/OSL.

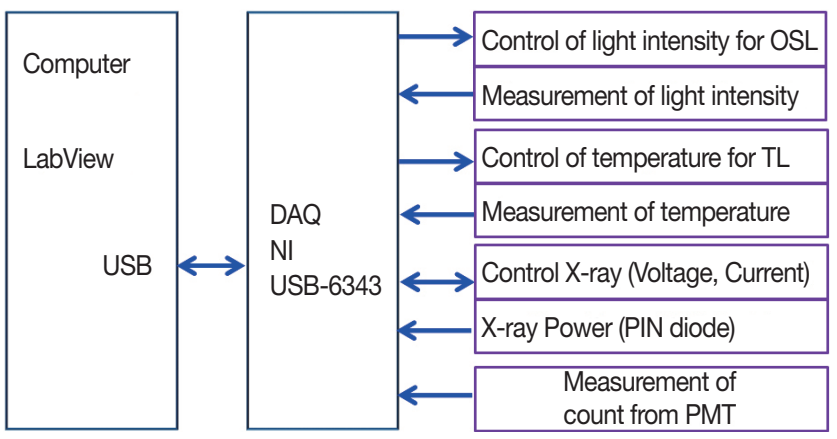

Fig. 4. Flowchart for controlling of RL/TL/OSL reader with PC.

\section{Results and Discussion}

To evaluate the performance of the developed RL/TL/OSL measuring system, RL/OSL was continuously measured. Using the $\mathrm{Al}_{2} \mathrm{O}_{3}: \mathrm{C}$ (diameter, $4.8 \mathrm{~mm}$; thickness, $1.0 \mathrm{~mm}$; and mass, $70 \mathrm{mg}$ ) specimen by Landaur, USA, RL/OSL were measured. The same specimens were used to take the measurements repeatedly, and after the end of each measurement, the amount of the stimulant light was set to its maximum and irradiated for 100 seconds to initiate the specimen. Next, RL/ TL was measured as same manner for the $\mathrm{LiF}: \mathrm{Mg}, \mathrm{Cu}, \mathrm{Si}$ (diameter, $4.5 \mathrm{~mm}$; thickness, $0.8 \mathrm{~mm}$; and mass, $30 \mathrm{mg}$ ) developed by the Korea Atomic Energy Research Institute [8, 9]. The same specimen was repeatedly used, and after the end of each measurement, the specimen was initiated at $260^{\circ} \mathrm{C}$ in an electric furnace for the next measurement.

\section{Output Characteristics of X-ray}

First, the output characteristics of the X-ray source were verified. The verification of the operation of the $\mathrm{X}$-ray source was conducted by outputting and monitoring the accelerated voltage and current in voltage form from the high voltage power supply of X-ray system. Meanwhile, $\mathrm{Al}_{2} \mathrm{O}_{3}$ :C shows $\mathrm{RL}$ phenomena if radiation is irradiated, which can be used to verify whether the $\mathrm{X}$-ray source is operational. The accelerated voltage of the X-ray source was set to $50 \mathrm{kV}$, and the current was varied from 0 to $200 \mu \mathrm{A}$ by $20 \mu \mathrm{A}$ step. This corresponds to the scope of 0 to $10 \mathrm{~W}$ by $1 \mathrm{~W}$ step if converted to the power of the X-ray source. The X-ray source was turned on for 10 seconds and turned off for 20 seconds so that it was

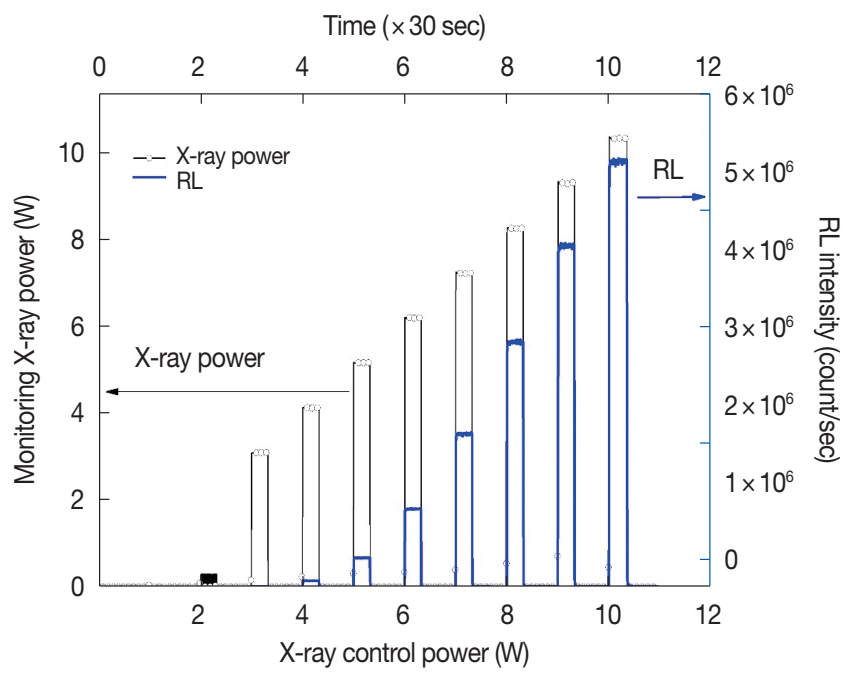

Fig. 5. RL curves for $\mathrm{Al}_{2} \mathrm{O}_{3}: \mathrm{C}$ irradiated by $1-10 \mathrm{~W}$ of $\mathrm{X}$-ray $50 \mathrm{kV}$. 


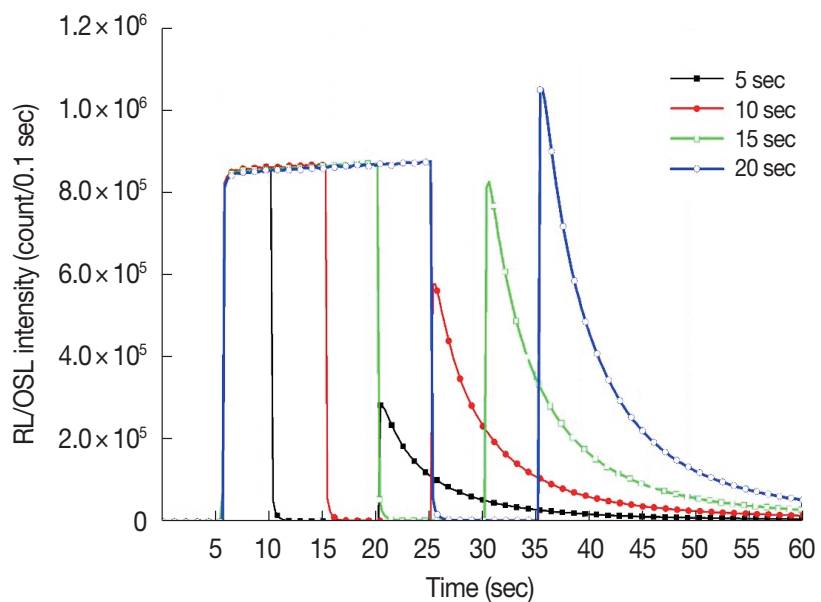

Fig. 6. $\mathrm{RL} / \mathrm{OSL}$ curves for $\mathrm{Al}_{2} \mathrm{O}_{3}: \mathrm{C}$ irradiated by $5-20$ seconds of $\mathrm{X}$ ray $50 \mathrm{kV}, 100 \mu \mathrm{A}$.

measured continuously for 330 seconds in total. The power values converted from the monitoring accelerated voltages and currents from the operation of the X-ray source as well as the $\mathrm{RL}$ luminescence curves of $\mathrm{Al}_{2} \mathrm{O}_{3}: \mathrm{C}$ are shown in Figure 5. Here, it can be confirmed that the X-ray source is operating stably at or over $2.5 \mathrm{~W}$. It can also be confirmed that as the output of the X-ray source increases, the RL luminescence curve of $\mathrm{Al}_{2} \mathrm{O}_{3}: \mathrm{C}$, the radiation also increases.

Figure 6 shows the continuous measurement of RL and OSL, whereas the X-ray source irradiates at $50 \mathrm{kV}$ and $100 \mu \mathrm{A}$ for 5 to 20 seconds. After 5 seconds of the measurement, the $\mathrm{X}$-ray source operates for a specified time. As X-ray is irradiated onto the specimen, RL is measured. Then, after 10 seconds, the stimulation light source is operated and OSL is measured. The RL signal gradually increases while the X-ray source runs, and the initial peak of OSL increases consistently as the exposure time increases, which leads to the increase of the cumulative exposure.

The RL curves from the RL/OSL measurement results, as shown in Figure 7, were used to assess the RL measurement performance against the accelerated voltage and current. The measurement of RL when the current of the X-ray source is set to $50 \mathrm{kV}, 100 \mu \mathrm{A}$ is shown in Figure 7A, and its operation time was maintained for 5-20 seconds while varying its accelerated voltage. The RL was measured when the X-ray source was in operation, and the RL curves maintained consistent.

As shown in Figure 7B of the dose response curve, the total luminescence shows a linear characteristic according to the dose. Such results were used to derive the linear function of the relation between the dose and the total luminescence.
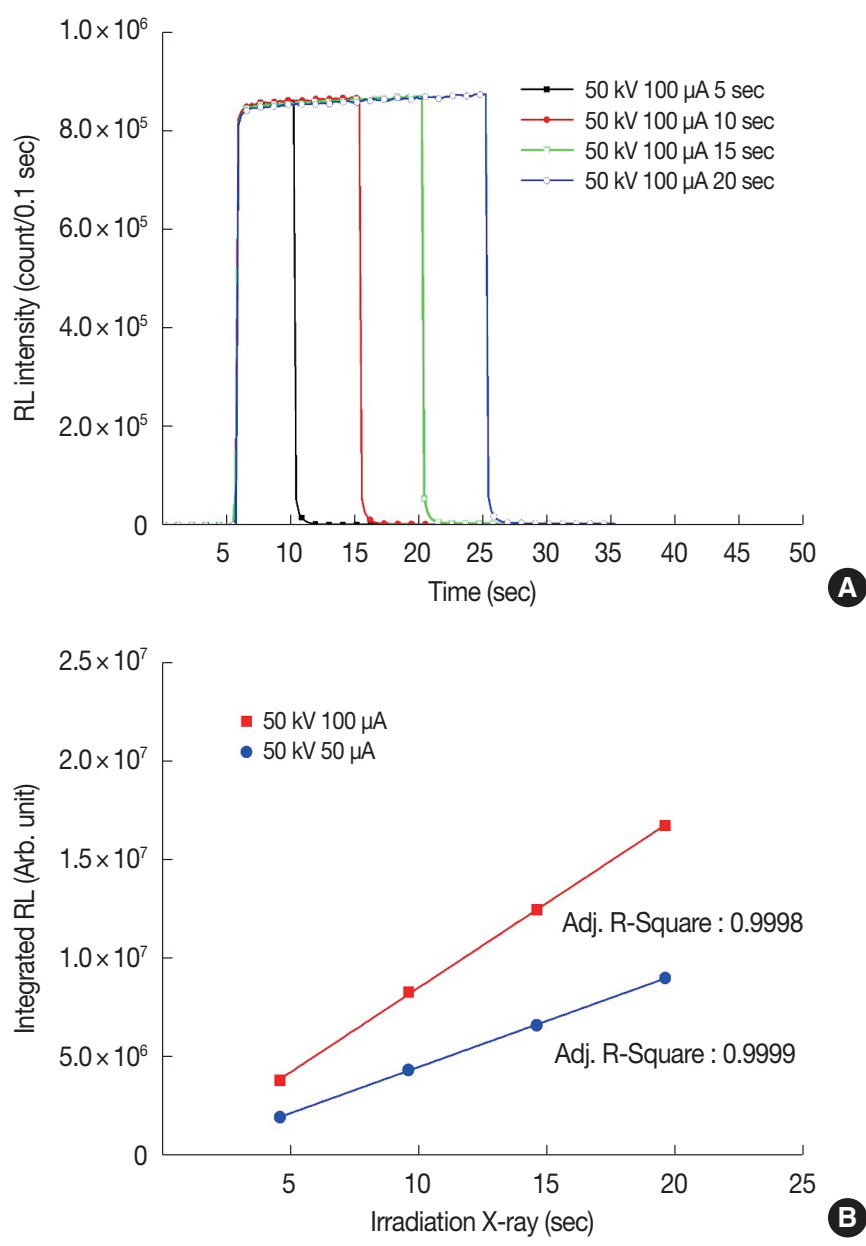

Fig. 7. (A) RL curves curves for $\mathrm{Al}_{2} \mathrm{O}_{3}: \mathrm{C}$ irradiated by 5-20 seconds of $X$-ray $50 \mathrm{kV}, 100 \mu \mathrm{A}$. (B) Dose response curve of the $R L$.

With the accelerated voltage at $50 \mathrm{kV}$ and the current at 50 $\mu \mathrm{A}, R^{2}=99.99 \%$, and with the current at $100 \mu \mathrm{A}, R^{2}=99.98 \%$, all of which shows a relatively linear characteristic.

\section{RL-OSL Measurement Characteristics}

The RL curves obtained from the RL/OSL measurement results, as shown in Figure 8, were used to assess the RL measurement performance against the various accelerated voltage and current for X-ray source. The measurement results of RL when the current of the X-ray source is set to 100 $\mu \mathrm{A}$ is shown in Figure 8A, and its operation time was maintained for 10 seconds while varying its accelerated voltage. When the X-ray source was in operation and the accelerated voltage increased from 30,40 , and $50 \mathrm{kV}$, the average RL increased from $2.773 \times 10^{5}$ count per 0.1 second to $6.424 \times 10^{5}$ and $8.596 \times 10^{5}$ count per 0.1 second, respectively. On the contrary, Figure $8 \mathrm{~B}$ shows the average RL for 10 seconds as the current of the X-ray source is 50 or $100 \mu \mathrm{A}$ and the accel- 

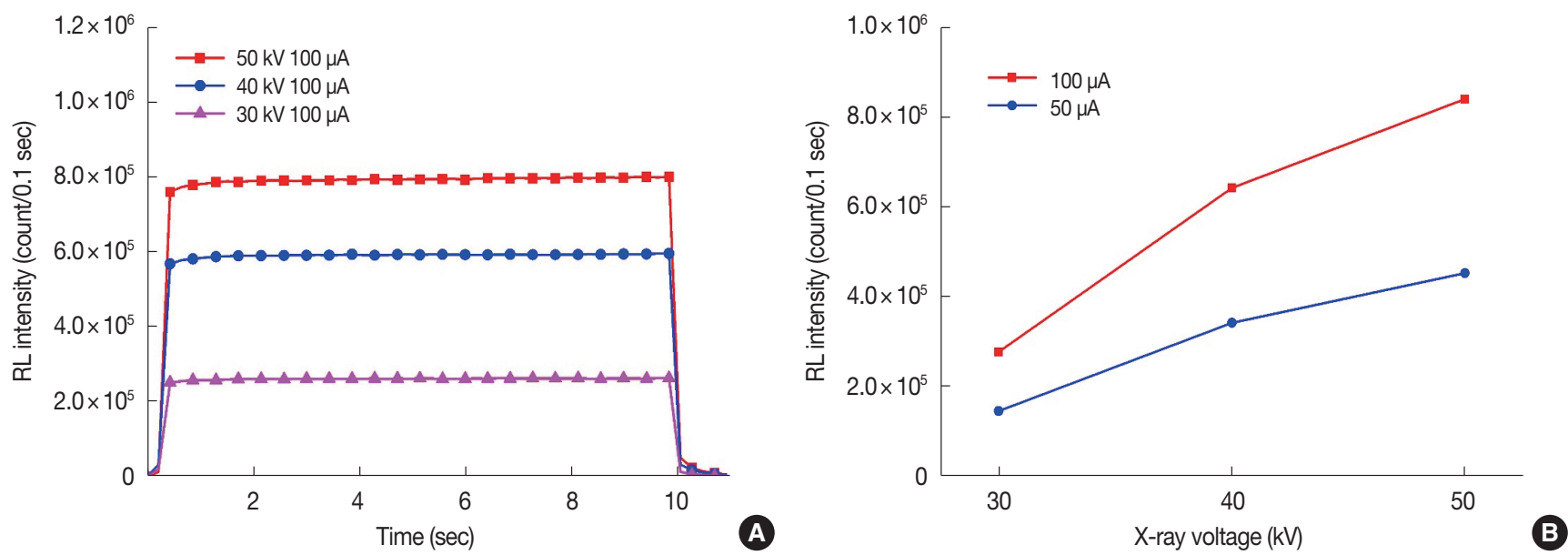

Fig. 8. (A) RL curves for $\mathrm{Al}_{2} \mathrm{O}_{3}: \mathrm{C}$ irradiated by 10 seconds of $\mathrm{X}$-ray 30,40 , and $50 \mathrm{kV}$ with $100 \mu \mathrm{A}$. (B) X-ray Voltage vs. average $\mathrm{RL}$ Intensity.
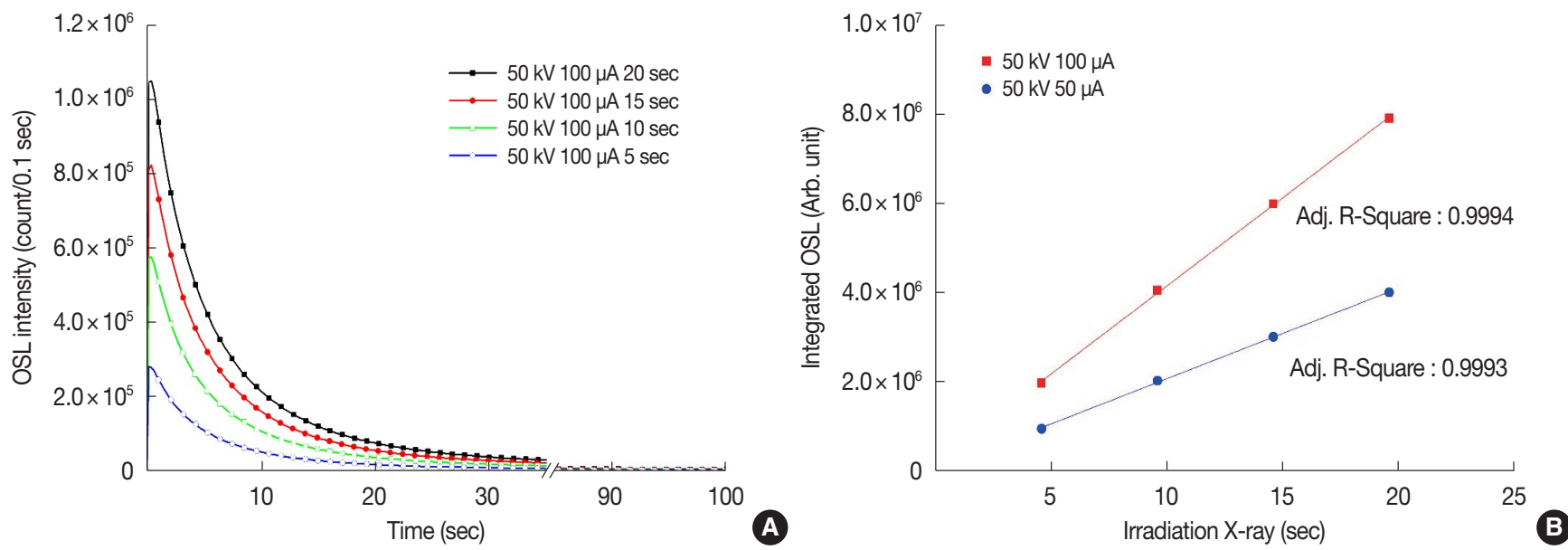

Fig. 9. (A) OSL curves curves for $\mathrm{Al}_{2} \mathrm{O}_{3}: \mathrm{C}$ irradiated by 5-10 seconds of $\mathrm{X}$-ray $50 \mathrm{kV}$ with $100 \mu \mathrm{A}$. (B) Dose response curve of the OSL.

erated voltage changes from 30,40 , and $50 \mathrm{kV}$, respectively. Due to an increase in the current, the average RL for $10 \mathrm{sec}-$ onds increased.

To assess the OSL measurement performance, the OSL curves from the RL/OSL measurement results, as shown in Figure 9, were used. Figure 9A shows the OSL when the current of the X-ray source is set to $100 \mu \mathrm{A}$ and the accelerated voltage was maintained at $50 \mathrm{kV}$ while the operation time of the source changed between 5 and 20 seconds. As the radiation of the X-ray source increased, the OSL signal also increased. As shown in Figure 9B of the dose response curve, the total luminescence for 100 seconds shows a linear characteristic according to the dose. Such results were used to derive the linear function of the relation between the dose and the total luminescence. With an accelerated voltage of $50 \mathrm{kV}$ and the current of $50 \mu \mathrm{A}, R^{2}=99.93 \%$, and with the current of $100 \mu \mathrm{A}, R^{2}=99.94 \%$, all of which shows a relatively linear characteristic. On the contrary, with the current at 100 $\mu \mathrm{A}$ and the accelerated voltage at $30 \mathrm{kV}, R^{2}=99.99 \%$, and with the accelerated voltage at $40 \mathrm{kV}, R^{2}=99.98 \%$.

\section{RL-TL Measurement Characteristics}

To investigate the RL/TL performance, $\mathrm{LiF}: \mathrm{Mg}, \mathrm{Cu}, \mathrm{Si}$ was irradiated at $50 \mathrm{kV}, 100 \mu \mathrm{A}$ for 5 seconds, and the heating rates were set 1,2 , and $5 \mathrm{~K} \cdot \mathrm{sec}^{-1}$ in the temperature range $20^{\circ} \mathrm{C}$ to $260^{\circ} \mathrm{C}$. Figure $10 \mathrm{~A}$ shows the continuous measurement results of $\mathrm{RL}$ and TL with a heating rate of $2 \mathrm{~K} \cdot \mathrm{sec}^{-1}$. The first section before 20 seconds was the RL measurement, and the second section after 20 seconds was the TL curves by the thermal stimulation. Meanwhile, Figure 10B shows that when TL glow curves were measured with different temperature increase rates, the position of the main peak increases from 496 to $509 \mathrm{~K}$ for temperature, and from $1.665 \times 10^{4}$ to $1.028 \times 10^{5}$ count per 0.1 second for the intensi- 

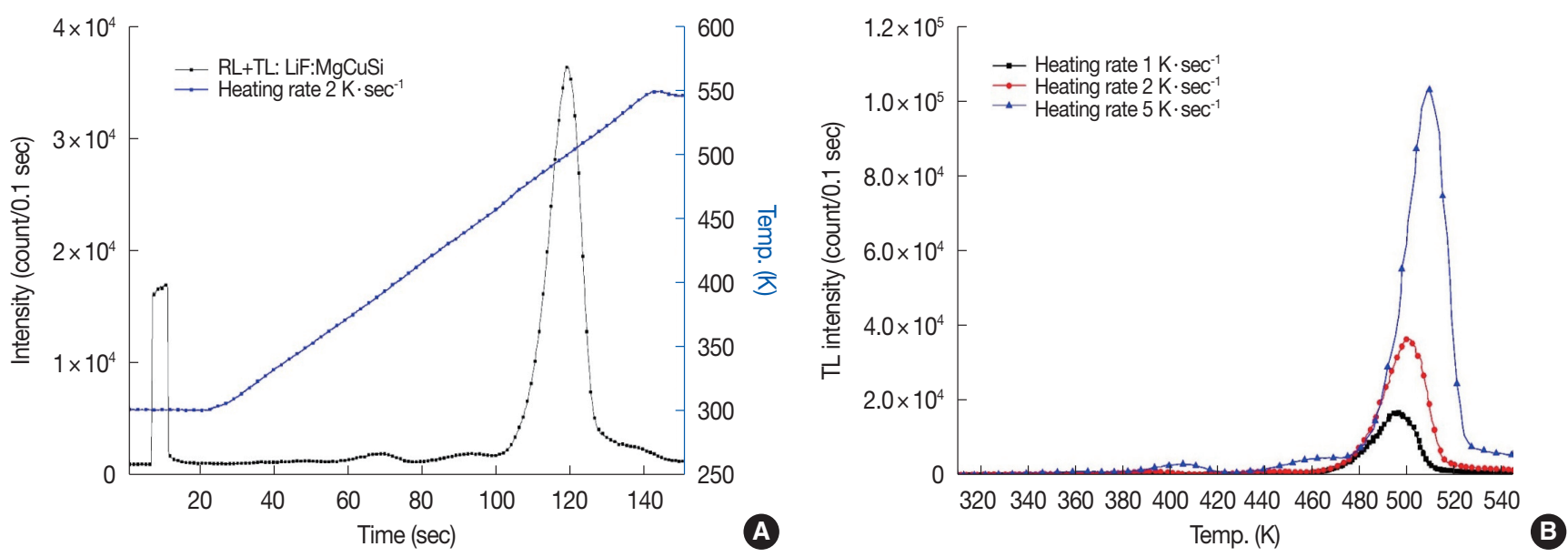

Fig. 10. RL/TL curves curves of LiF:Mg,Cu,Si irradiated by 5 seconds of X-ray $50 \mathrm{kV}, 100 \mu \mathrm{A}$. (A) $2 \mathrm{~K} \cdot \mathrm{sec}^{-1}$ and (B) TL curves curves of LiF:Mg,Cu,Si.

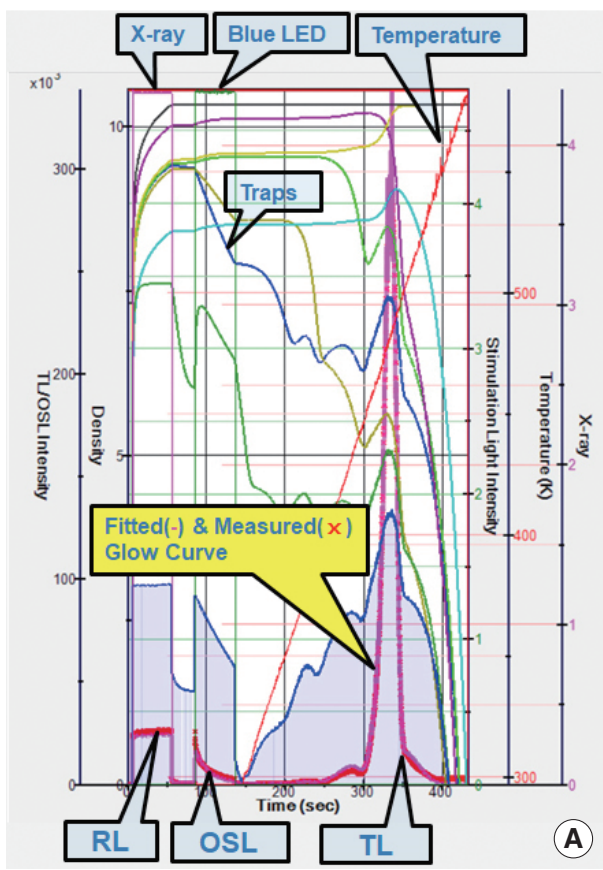

Material Int M. Add Electron Irap Add Hole Ira

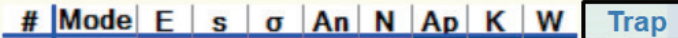

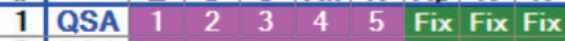

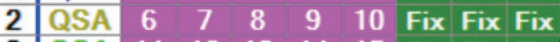

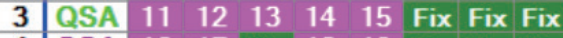
4 QSA $16 \quad 17$ Fix 18 19 19 Fix Fix Fix 5 QSA $20 \quad 21$ Fix $22 \quad 23$ Fix Fix Fix 6 QSA Fix Fix Fix 2425 Fix Fix Fix \begin{tabular}{l|llllllll}
7 & QSA & 26 & 27 & Fix & 28 & 29 & Fix Fix Fix
\end{tabular} \# Mode $\mathbf{E}|\mathbf{s}| \boldsymbol{\sigma}|\mathbf{A g}| \mathbf{M}|\mathbf{A m}| \mathbf{K} \mid \mathbf{W} \quad \mathbf{R C}$ 1 QSA 2 QSA Fix Fix Fix $\begin{array}{llllll}33 & 34 & 35 & \text { Fix Fix }\end{array}$ Exposure (n0, m0. nc0)

what It $\min \mid$ value $\quad \max$

\begin{tabular}{l|l|l|l}
\hline E1-n1 Fix 1.000000E-001 & $1.000000 \mathrm{E}-001$ & $1.000000 \mathrm{E}+010$
\end{tabular}

E1-n2 Fix 1.000000E-001 1.000000E-001 1.000000E+010

E1-n3 Fix 1.000000E-001 1.000000E-001 1.000000E+010

E1-n4 Fix 1.000000E-001 1.000000E-001 1.000000E+010

E1-n5 Fix 1.000000E-001 1.000000E-001 $1.000000 \mathrm{E}+010$

E1-n6 Fix 1.000000E-001 1.000000E-001 $8.776469 \mathrm{E}+008$

E1-n7 Fix 1.000000E-001 1.000000E-001 $1.000000 \mathrm{E}+010$

\begin{tabular}{llllll|l}
\hline$E 1-m 1$ & 36 & $6.406095 E+006$ & $1.866993 E+011$ & $2.051577 E+013$ \\
\hline
\end{tabular}

\begin{tabular}{l|l|l|l|l|l}
\hline E1-m2 37 & $1.411879 \mathrm{E}+004$ & $1.548007 \mathrm{E}+008$ & $2.304822 \mathrm{E}+012$ \\
\hline
\end{tabular}

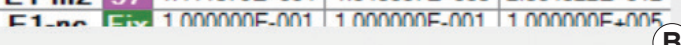

Fig. 11. RL/OSL/TL curves LiF:Mg,Cu,Si irradiated by X-ray 50 kV, $100 \mu \mathrm{A}$.

ty as the temperature increase rate rises. These luminescence curves measured can be used to analyze the properties related to the radiation, thermal and optical stimulation characteristics of the trapping level and recombination center (RC) using LumiAnal 2016 glow curves measured as such can be used to [10].

Generic analysis programs use the OTOR model, which restricts each peak of the luminescence curve centered on one trap and one recombination center. However, the proposed system extends this further using the multi trap multi recombination center model where there may be several traps for electrons and several. It can help us better understand the luminescence phenomena as electrons and holes can move conduction bands, valence bands, several traps, and RCs through the radiation stimulation as well as the thermal and optical stimulation. An example of the analysis process that uses the measurement results of the luminescence curves and LumiAnal 2016 is shown in Figure 11. The specimen used was $\mathrm{LiF}: \mathrm{Mg}, \mathrm{Cu}, \mathrm{Si}$, which showed the measurement of the RL/OSL/TL luminescence curve. The measurement procedures are as follows: first, RL was measured while X-ray was irradiated for 50 seconds at $50 \mathrm{kV}, 100 \mu \mathrm{A}$. 
Optical stimulation was added in the middle of the process for the 50 seconds to measure OSL, and later TL was sequentially measured with the thermal stimulation condition where the heating rate was $1 \mathrm{~K} \cdot \mathrm{sec}^{-1}$ between $20^{\circ} \mathrm{C}$ and $260^{\circ} \mathrm{C}$. During the analysis process, seven traps and two RCs were introduced to analyze the luminescence curve. The measured and analyzed luminescence curve is shown in Figure 11A. The electron concentration of the traps and the hole concentration of the RCs were indicated with different colored curve, and each stimulation was also expressed in its own way. The introduced seven traps and two RCs were lined up, and each parameter and initial value to be analyzed are shown in Figure 11. At each parameter, 'Fix' was the fixed value, and the one expressed in numbers is to be analyzed. Using them, the trend in the change of the electron and hole concentration based on each stimulation could be verified by changing corresponding parameters. In other words, when the X-ray was irradiated, electrons are filled up in the traps so the electron concentration increased, and some moved toward the RCs to produce the RL luminescence curves. Furthermore, in the optical stimulation process, the electron concentration in the three traps decreased, producing the OSL luminescence curves. Finally, the electron concentration decreased sequentially based on the thermal stimulation, producing the TL luminescence curves. Therefore, the process in which the overall contribution of the electron concentration in the traps and the hole concentration in the RCs to the luminescence in time can be determined by the analysis process [11].

\section{Conclusion}

In this study, an RL/TL/OSL integrated measuring system that can consistently measure the RL, TL, and OSL under the identical condition was developed. For X-ray irradiation, this system used the $50 \mathrm{kV}$ MAGNUM X-ray Tube by Moxtek as the X-ray source. A $1 \mathrm{~mm}$-thick aluminum panel was used to block the energy below $20 \mathrm{kV}$, and the dose irradiated to the specimen was measured with a Silicon PIN photodiode. For the thermal stimulation, this system used the sample substrate as the heating element. The high brightness blue LED was used as the stimulating light for OSL and the measurement efficiency was also improved by optimizing the filter combination. Luxeon V-type high brightness blue LED with the bandwidth of $470 \pm 25 \mathrm{~nm}$ was used as the stimulating light. Thus, sufficient amount of luminance can be acquired by using three of them. Also, since the signals obtained from $\mathrm{RL} / \mathrm{TL} / \mathrm{OSL}$ are weak, these signals were measured by using the count mode of PMT. The voltage, current, and exposure time of the X-ray source were controlled by a control program, and the thermal stimulation temperature was controlled by changing the temperature. The amount of the stimulant luminance was controlled to measure CW-OSL and LM-OSL. Various measurements and analyses can be conducted using the proposed system. First, $\mathrm{Al}_{2} \mathrm{O}_{3}: \mathrm{C}$ was used to measure $\mathrm{RL} /$ OSL and RL and OSL were analyzed. The measurement of RL was conducted by maintaining the current of the X-ray source at $100 \mu \mathrm{A}$ and its operation time was 10 seconds, while the accelerated voltage changed from 30 to $50 \mathrm{kV}$. When the X-ray source was operational, RL was maintained consistent, and as the accelerated voltage increased from 30 to $40 \mathrm{kV}$ and $50 \mathrm{kV}$, the average $\mathrm{RL}$ for 10 seconds increased from $2.773 \times 10^{5}$ count per 0.1 second to $6.424 \times 10^{5}$ and $8.596 \times 10^{5}$ count per 0.1 second, respectively. To confirm the dose response characteristics, the integrated intensity of OSL measured for 100 seconds was used, and the results showed a linear characteristic by dose. With the accelerated voltage at $50 \mathrm{kV}$ and the current at $50 \mu \mathrm{A}, R^{2}=99.93 \%$, and with the current at $100 \mu \mathrm{A}, R^{2}=99.94 \%$, showing a relatively linear property.

The RL/TL measurement characteristics were measured by changing the temperature increase rate, using $\mathrm{LiF}: \mathrm{Mg}, \mathrm{Cu}$, Si. The stimulating temperature by the temperature increase rate showed a consistent rise. Furthermore, the RL characteristics showed identical luminescence in the repeated tests. The TL measurement part showed that as the temperature increase rate rises from 1 to $5 \mathrm{~K} \cdot \mathrm{sec}^{-1}$, the location of the main peak increased from 496 to $509 \mathrm{~K}$ for temperature and from $1.665 \times 10^{4}$ to $1.028 \times 10^{5}$ count per 0.1 second for intensity. In this study, the integrated measurement system, which can relatively easily, inexpensively, and efficiently measure the signals from RL/TL/OSL, has been developed using the universal USB-DAQ and LabView program and we have confirmed the excellent performance through the analysis of luminescence curves.

\section{Acknowledgements}

This work was supported by Development Fund Foundation, Gyeongsang National University, 2016. 


\section{References}

1. Chen R, Pagonis V. Thermally and Optically Stimulated Luminescence. 1st Ed. East Sussex. Wiley. UK. 2011;7-28.

2. Huntley DJ, Godfrey-Smith DI, Thewalt MLW. Optical dating of sediments. Nature. 1985;313:105-107.

3. Yukihara EG, McKeever SWS. Optically Stimulated Luminescence: Fundamentals and Applications. 1st Ed. East Sussex. Wiley. UK. 2011;14-33.

4. McKeever SWS. Thermoluminescence of Solids. 1st Ed. Cambridge. Cambridge University Press. Cambridge, UK. 1985;40-63.

5. Damkjær SMS, Andersen CE, Aznar MC. Improved real-time dosimetry using the radioluminescence signal from $\mathrm{Al}_{2} \mathrm{O}_{3}: \mathrm{C}$. Radiat. Meas. 2008;43(2-6):893-897.

6. Park CY, Chung KS, Lee JD, Chang IS, Lee JI, Kim JL. Development of OSL dosimetry reader. J. Radiat. Prot. Reas. 2012;37(1): 10-15.

7. Park CY, Chung KS, Lee JD, Chang IS, Lee JI, Kim JL. Develop- ment of thermoluminescence and optical stimulated luminescence measurements system. J. Radiat. Prot. Reas. 2015;40(1): 46-54.

8. Lee JI, Kim JL, Yang JS, Pradhan AS, Kim BH, Chung KS, Choe HS. Dual-step thermal treatment for the stability of glow curve structure and the TL sensitivity of the newly developed LiF:Mg, Cu,Si. Radiat. Meas. 2007;42(4-5):597-600.

9. Pradhan AS, Lee JI, Kim JL, Chung KS, Choe HS, Lim KS. TL glow curve shape and response of $\mathrm{LiF}: \mathrm{Mg}, \mathrm{Cu}, \mathrm{Si}$-Effect of heating rate. Radiat. Meas. 2008;43(2-6):361-364.

10. Chung KS, Lee JI, Kim JL. A computer program for the deconvolution of the thermoluminescence glow curves by employing the interactive trap model. Radiat. Meas. 2012;47(9):766-769.

11. Chung KS, Park CY, Lee JI, Kim JL. An algorithm for the integrated deconvolution of radioluminescence and thermally/optically stimulated luminescence glow curves. Radiat. Meas. 2015;79: 7-12. 